\title{
Dynamics of Pro- and Anti-inflammatory Cytokines in Experimental Animals with Non-alcoholic Fatty Liver Disease Under Conditions of Hypobaric Hypoxia
}

\author{
AQ1 Nurgul Toktogulova ${ }^{1 *}$ (D), Rustam Tuhvatshin ${ }^{2}$ (D), Elmira Mainazarova ${ }^{3}$ (D)
}

${ }^{1}$ Department of Therapy, Pediatrics and Dentistry Specialization, Kyrgyz-Russian Slavic University, Bishkek, Kyrgyzstan; ${ }^{2}$ Department of Pathological Physiology, Doctor of Medical Sciences, Kyrgyz State Medical Academy, Bishkek, Kyrgyzstan; ${ }^{3}$ Department of Epidemiology and Immunology, Kyrgyz-Russian Slavic University, Bishkek, Kyrgyzstan

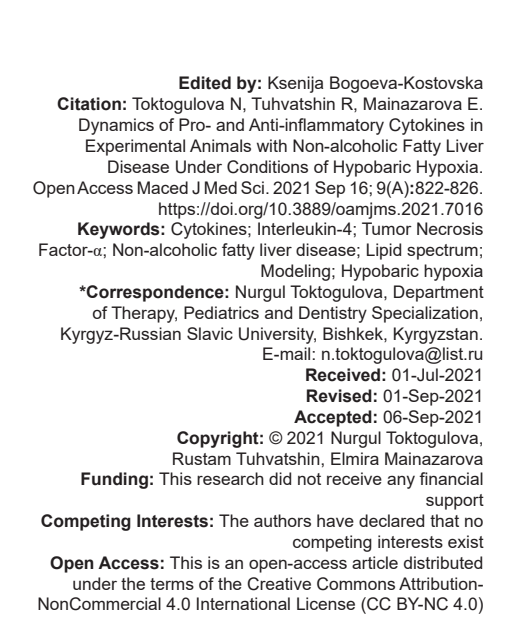

\begin{abstract}
AIM: The aim of the study was to study the level of pro- and anti-inflammatory cytokines, tumor necrosis factor-alpha $(\mathrm{TNF}-\alpha)$, and interleukin 4 (IL-4), on a model of non-alcoholic fatty liver disease (NAFLD) in experimental animals under conditions of low mountains and hypobaric hypoxia.

METHODS: The study was carried out on 180 male Wistar rats, which were divided into control and experimental groups. The rats of the control group were on a standard diet. NAFLD was modeled by keeping animals on a diet (Ackermann et al., 2005) rich in fructose and fat in conditions of low mountains and hypobaric hypoxia (in a pressure chamber $6000 \mathrm{~m}$ above sea level) for 35 and 70 days. Total cholesterol (TC) and lipid spectrum, pro- and antiinflammatory cytokines were determined in all groups of animals.

RESULTS: The activity of pro- and anti-inflammatory cytokines in the main group during the rise of animals in the pressure chamber increased statistically significantly on the $35^{\text {th }}$ day in comparison with the low-altitude group by more than 2 times, and on the $70^{\text {th }}$ day of staying at the high-altitude did not have convincing differences from the lowaltitude group. The cytokine index (TNF- $\alpha / \mathrm{IL}-4)$ of animals in conditions of hypobaric hypoxia on a fructose enriched diet increased by more than 1.5 times after 5 weeks, staying at an altitude for 10 weeks led to a decrease of TNF- $\alpha / \mathrm{IL}-4$ in relation to the low-mountain group, in which the opposite picture was observed - a tendency towards an increase in TNF- $\alpha /$ IL-4. IL-4 and TNF- $\alpha$ levels were statistically significantly correlated with lipid metabolism disorders.

CONCLUSION: NAFLD in animals on a special diet enriched with fructose under conditions of hypobaric hypoxia leads to deeper disturbances in the system of pro- and anti-inflammatory cytokines and the lipid spectrum.
\end{abstract}

\section{Introduction}

Non-alcoholic fatty liver disease (NAFLD) is the most common chronic liver disease worldwide, affecting nearly $25 \%$ of adults. Despite its high prevalence, only a small number of patients develop inflammation, and then fibrosis and chronic inflammation of the liver, while many of them are dominated by simple steatosis [1]. In this context, it is extremely important to fully understand the mechanisms underlying the development of NAFLD and non-alcoholic steatohepatitis (NASH). Despite advances in this area, knowledge about the pathogenesis of NAFLD is still incomplete. The multiple shock hypotheses consider various influences such as insulin resistance, hormones secreted by adipose tissue, nutritional factors, gut microbiota, genetic, and epigenetic factors [1]. Most acute and chronic liver diseases are characterized by inflammatory processes with increased expression of various pro- and antiinflammatory cytokines in the liver. These cytokines are the main causes of inflammatory liver diseases, often leading to fibrosis and cirrhosis. Under physiological conditions, the generation of constitutive cytokines in the liver is absent or minimal. However, pathological stimuli, such as lipid accumulation, prompt liver cells to produce these inflammatory molecules. Cytokines may play an active role in the development and potential progression of NAFLD by stimulating liver inflammation, cell necrosis and apoptosis, and the progression of fibrosis. However, they are also required for liver regeneration after injury [2], [3]. Proinflammatory cytokines such as interleukin 1 (IL-1), IL-6 and tumor necrosis factor-alpha (TNF- $\alpha$ ) play an important role in the pathogenesis of NAFLD. These cytokines play a crucial role in the development of insulin resistance, which is a key factor in the pathogenesis of NAFLD. TNF-a was first described pro-inflammatory cytokine associated with obesity and insulin resistance [3]. Koca et al. consider that adipose tissue is an important source of inflammation caused by obesity, in particular due to the expression of TNF- $\alpha$, which can cause inflammation and insulin resistance. Moreover, the use of antibodies against TNF- $\alpha$ in an experimental model of NASH reduced inflammation, necrosis, and fibrosis in rats [4], [5]. Some studies 


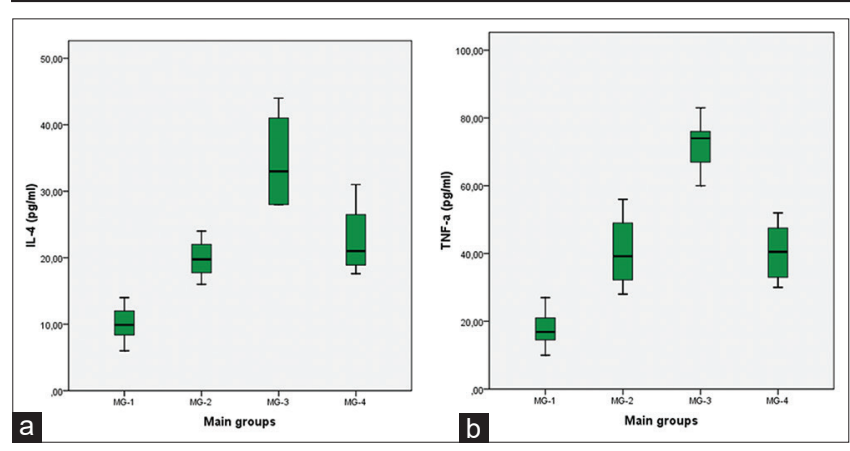

Figure 1: Concentrations of IL-4 (a) and TNF- $\alpha$ (b) in the experimental groups

describe a pronounced increase in the activity of the pro-inflammatory cytokine TNF- $\alpha$ with a significant suppression of the body's protective potential in the form of a decrease in the activity of anti-inflammatory cytokines (IL-2 and IL-4) [6], and it was revealed that inflammation underlies the development of metabolic disorders and their complications [7]. The state of anti-inflammatory ILs has also been studied from the standpoint of the combination of type 2 diabetes mellitus with NAFLD. Another study describes changes in the level of cytokines and subpopulation composition of lymphocytes, which play a role in the intensity and direction of immunopathological reactions (increase in INF- $\gamma$, decrease in IL-4, and change in the content of NK cells), the intensity of proliferation of connective tissue and the degree of fibrosis (increase in IL-10) [8]. There is little data in the literature on the course of NAFLD in high altitude conditions. In high altitude conditions, in addition to the action of the main factor a decrease in the partial pressure of oxygen, a number of other factors affect the body: Daily temperature fluctuations, increased radiation of the ultraviolet part of the spectrum, etc. The study of changes in the body under the influence of these factors is of great importance. There are no publications on the association of IL-4 and TNF- $\alpha$ with NAFLD developed in high altitude conditions. Therefore, the study of violations directly caused by the lack of oxygen in the experiment, it was decided to carry out on the model of hypoxia caused in a ventilated pressure chamber at a constant temperature. It is known that liver cells are in second place after brain cells in sensitivity to hypoxia. New publications showing the protective effect of chronic high-altitude hypoxia on the progression of NAFLD began to arrive from China only in July 2020 [9]. Thus, the aim of the study was to study the levels of pro- and anti-inflammatory cytokines (IL-4 and TNF- $\alpha$ ) in a model of experimental animals with NAFLD under conditions of low mountains and hypobaric hypoxia. In the course of the experiment, the following tasks were solved: To model fatty hepatosis in low- and highmountain groups of animals; to study the correlation of the level of cytokines with the lipid spectrum.

\section{Methods}

The study was carried out on 180 male Wistar rats weighing 150-200 $\mathrm{g}$ at the time of inclusion in the experiment. Before the start of the experiment, the research plan, standardized procedures and accompanying documentation were ethically reviewed and approved by the Ethics Committee of the Research and Production Association "Preventive Medicine" of the Ministry of Health of the Kyrgyz Republic (extract No. 6 dated October 8, 2019). The rats were randomly assigned to three control (20 animals in each group) and four experimental groups (30 animals in each group). The rats of the control group were kept on a standard diet (food "Eshka," Russia, total caloric content of $3000 \mathrm{kcal} / \mathrm{kg}$ ). NAFLD was modeled by the formation of metabolic syndrome in the experimental group (Ackermann et al. 2005). During the study period, the animals received food containing $20.7 \%$ proteins (casein), $5 \%$ fats (beef lard), 60\% carbohydrates (fructose), $8 \%$ cages, $5 \%$ minerals, and $1 \%$ vitamins [10]. Feeding was carried out 2 times a day. This model allows in the shortest possible time to reproduce NAFLD with all pathogenetic aspects: Hypercholesterolemia, triglyceridemia, and insulin resistance. The role of a fructose-rich diet in inflammation is well-known [1], [11]. Ectopic fat accumulation increases the vulnerability of hepatocytes to cellular stress, which initiates the inflammatory process [12]. In parallel, cellular stress can be exacerbated by inflammation caused by the activation of toll-like receptor 4 (TLR4) in Kupffer cells, since fructose promotes the synthesis of saturated fatty acids such as palmitate, which are able to activate TLR4 receptors in liver Kupffer cells [13]. Activation of the TLR4/inducible nitric oxide synthase/ NF $\kappa B$ pathway induces oxidative stress in hepatocytes through the production of pro-inflammatory cytokines such as TNF- $\alpha$ by Kupffer cells. These phenomena are supported by lipid-induced increase in the proportion of "normal" pro-inflammatory M1 macrophages compared to "alternative" anti-inflammatory M2 macrophages [14].

Animals were divided into three control groups of 20 animals each (CG) and 4 main groups of 30 animals each (MG): Control 1 " $(n=20)$ - intact, healthy rats kept in low mountains (Bishkek, $800 \mathrm{~m}$ above sea level) on a standard diet; "Control 2" ( $n=20)$ - hypobaric hypoxia (6000 $\mathrm{m}$ above sea level in a pressure chamber, daily exposure for $6 \mathrm{~h}$ for 5 weeks) on a standard diet [15]; "Control 3" ( $n=20)$ - hypobaric hypoxia (6000 m above sea level in a pressure chamber, $6 \mathrm{~h}$ - 10 weeks) on a standard diet; "Main group 1" $(n=30)$ - low-mountain group of rats for 5 weeks on a diet enriched with fructose (FED) [9]; "Main group 2" $(n=30)$ - low-mountain group of rats on FED for 10 weeks; "Main group 3" $(n=30)$ - a group of rats on FED under conditions of 
hypobaric hypoxia for 5 weeks; and "Main group 4" $(n=30)$ - a group of rats on FED under conditions of hypobaric hypoxia for 10 weeks. Blood sampling for analysis in groups of animals MG-1 and MG-3 groups was carried out on the $35^{\text {th }}$ day, and in the MG-2 and MG-4 groups on the $70^{\text {th }}$ day from the beginning of observations. In all groups of animals, the following parameters were determined: Total cholesterol (TC), Low-density lipoprotein (LDL) cholesterol, high-density lipoprotein (HDL) cholesterol, triglycerides (TG), and pro- and anti- inflammatory cytokines (TNF- $\alpha$ and IL-4). After clotting, the blood samples were centrifuged at approximately $1000 \mathrm{rpm}$ for $10 \mathrm{~min}$ and the serum was separated. Serum samples for IL-4 and TNF- $\alpha$ were aliquoted $(250-500 \mu \mathrm{L})$ to avoid repeated freeze-thaw cycles and stored frozen at $-70^{\circ} \mathrm{C}$. Cytokine levels were determined using an enzyme-linked immunosorbent assay kit based on a solid-phase "sandwich" variant using mono- and polyclonal antibodies to TNF- $\alpha$ and IL-4 in the laboratory of the Research Institute of Molecular Biology and Medicine at the Research Center of Cardiology and Therapy named after $M$. Mirrakhimov. The results were analyzed using the SPSS 16.0 statistical software package for Windows. To assess the probabilities that the analyzed samples belong to general populations with a normal distribution, the Kolmogorov-Smirnov test was used. Taking into account the normal distribution of sample data $(p>0.05)$, Student's t-test was used for comparison. The significance of differences between the groups was determined by nonparametric statistical methods, the results of descriptive processing were presented as the mean and the error of the mean $(\mathrm{M} \pm \mathrm{m})$. The Pearson correlation test was used to see the relationship between variables. A $p<0.05$ was considered statistically significant at the $95 \%$ confidence level.

\section{Results and Discussion}

Data on the content of cytokines in the blood in the studied groups are presented in Table 1. It should be noted that the control pressure chamber group ("CG- 2") had high levels of IL-4 and TNF- $\alpha$ compared to the control low-mountain group ("CG-1") with $p<0.05$. The activity of cytokines in animals with rise significantly increased after 5 weeks. Thus, on the $35^{\text {th }}$ day of stay at altitude, the content of IL-4 increased (from $27.5 \pm 5.5$ to $34.5 \pm 2.8 \mathrm{pg} / \mathrm{ml}$ and TNF- $\alpha$ from $49.5 \pm 2.5$ to $72.3 \pm 3.2$ ) against the low-mountain group by a diet rich in fructose and fat $-9,9 \pm 0.6$ and $17.8 \pm 1.1$, respectively. After 10 weeks of staying at an altitude in animals ("MG-4"), the levels of pro- and anti-inflammatory cytokines did not have statistically significant differences from the low-altitude group, which were also on FED ("MG-2") ( $p$ > 0.05) (Figure 1).

The balance between pro- and antiinflammatory cytokines plays a key role in the development of NAFLD [2]. In this context, the ratio of pro- and anti-inflammatory cytokines was investigated in all studied groups. There was a tendency towards higher levels of TNF- $\alpha / \mathrm{IL}-4$ in the blood in highaltitude animals on FED for 35 days $(2.2 \pm 0.2 \mathrm{pg} / \mathrm{ml})$ compared with the low-altitude group on an identical diet $(1.8 \pm 0.1 \mathrm{pg} / \mathrm{ml})$. After 10 weeks of staying at an altitude in the main group (MG-4), a decrease in the cytokine index was observed in relation to the low mountain group - MG-2 (1.8 \pm 0.1 vs. $2.0 \pm 0.1 \mathrm{pg} / \mathrm{ml})$.

Here, MG-1 and MG-2 groups - groups on a special diet on the $35^{\text {th }}$ and $70^{\text {th }}$ days; MG 3 and MG 4 - pressure chamber groups on a special diet on the $35^{\text {th }}$ and $70^{\text {th }}$ days.

As shown in Table 2, the TC and LDL cholesterol of the main group of animals (MG-3) in the pressure chamber increased statistically significantly at 5 weeks. Thus, on the $35^{\text {th }}$ day of stay at altitude, the content of TC increased from $3.7 \pm 0.1$ to $6.6 \pm 0.8 \mathrm{mmol} / \mathrm{L}$ compared with the low-mountain group (MG-1) $(1.2 \pm 0.1$ and $1.7 \pm 0.2 \mathrm{mmol} / \mathrm{L}$, respectively). The same tendency in the level of LDL is observed at 5 weeks, an increase from $2.5 \pm 0.1$ to $4.0 \pm 0.5 \mathrm{mmol} / \mathrm{L}$ in MG-3 versus MG-1 $0.2 \pm 0.1$ and $0.2 \pm 0.1 \mathrm{mmol} / \mathrm{L}$, respectively, with $p<0.001$. At week 10 in the pressure chamber in the MG-4 group, the levels of TC and LDL remained statistically significantly high, although there was a downward trend. On the contrary, in the lowmountain group, there was a positive trend in both indicators. It should be noted that the initial levels of TC and LDL in the high mountain group were high compared to the low mountain group. The mean values of TG and HDL were higher in the MG-3 group (3.1 [0.6-6.2] and $1.2 \pm 0.1$, respectively) compared with the control (0.74 [0.72- 0.76] and $0.9 \pm 0.1$, respectively) with $p<0.05$. The values of the coefficient of atherogenicity in all groups consistently demonstrated a similar trend.

Taking into account the important role of immune inflammation and cholesterol metabolism

Table 1: Content of pro- and anti-inflammatory cytokines in experimental animals with non-alcoholic fatty liver disease, $\mathrm{pg} / \mathrm{ml}, \mathrm{M} \pm \mathrm{m}$

\begin{tabular}{|c|c|c|c|c|c|c|c|c|}
\hline Indicator & $\frac{\text { CG-1 }(n=20)}{1}$ & $\frac{C G-2(n=20)}{2}$ & $\frac{C G-3(n=20)}{3}$ & $\begin{array}{l}\text { MG-1 }(n=30) \\
4\end{array}$ & $\frac{M G-2(n=30)}{5}$ & $\begin{array}{l}\text { MG-3 }(n=30) \\
6\end{array}$ & $\begin{array}{l}\text { MG-4 }(n=30) \\
7\end{array}$ & $p$-value \\
\hline $\mathrm{IL}-4 \mathrm{pg} / \mathrm{ml}$ & $4.4 \pm 0.4$ & $27.5 \pm 5.5$ & $19.0 \pm 1.0$ & $9.9 \pm 0.6$ & $19.8 \pm 1.6$ & $34.5 \pm 2.8$ & $22.6 \pm 1.7$ & $\begin{array}{l}P_{1-2}<0.05 \\
P_{46}<0.001\end{array}$ \\
\hline TNF- $\alpha \mathrm{pg} / \mathrm{ml}$ & $6.9 \pm 0.7$ & $49.5 \pm 2.5$ & $36.5 \pm 2.5$ & $17.8 \pm 1.1$ & $40.6 \pm 5.8$ & $72.3 \pm 3.2$ & $40.5 \pm 2.9$ & $\begin{array}{l}\mathrm{P}_{1-2}^{4-6}<0.05 \\
\mathrm{P}_{4-6}<0.001\end{array}$ \\
\hline TNF- $\alpha /$ IL-4 & $1.6 \pm 0.2$ & $1.8 \pm 0.3$ & $1.9 \pm 0.1$ & $1.8 \pm 0.1$ & $2.0 \pm 0.1$ & $2.2 \pm 0.2$ & $1.8 \pm 0.1$ & $\begin{array}{l}P_{1-2}^{4-6}>0.05 \\
P_{4-6}>0.05 \\
P_{5-7}>0.05\end{array}$ \\
\hline
\end{tabular}


Table 2: Lipid spectrum in experimental animals with NAFLD, $M \pm m$

\begin{tabular}{|c|c|c|c|c|c|c|c|c|}
\hline Indicator & CG-1 $(n=20)$ & CG-2 $(n=20)$ & CG-3 $(n=20)$ & MG-1 $(n=30)$ & MG-2 $(n=30)$ & MG-3 $(n=30)$ & MG-4 $(n=30)$ & $p$-value \\
\hline & 1 & 2 & 3 & 4 & & & & \\
\hline $\mathrm{TC} \mathrm{mmol} / \mathrm{l}$ & $1.2 \pm 0.1$ & $3.7 \pm 0.1$ & $3.2 \pm 0.1$ & $1.7 \pm 0.2$ & $2.1 \pm 0.1$ & $6.6 \pm 0.8$ & $5.3 \pm 0.4$ & $\begin{array}{l}\mathrm{P}_{1-2 \cdot, 3}<0.05 \\
\mathrm{P}_{4-6}<0.001 \\
\mathrm{P}_{5-7}<0.001\end{array}$ \\
\hline TG mmol/l & $0.59 \pm 0.1$ & $0.74 \pm 0.1$ & $0.59 \pm 0.1$ & $1.4 \pm 0.3$ & $0.7 \pm 0.2$ & $3.1 \pm 0.8$ & $1.1 \pm 0.1$ & $\begin{array}{l}\mathrm{P}_{1-1,2,3}>0.05 \\
\mathrm{P}_{4-6}>0.05 \\
\mathrm{P}_{-6,}<0.05\end{array}$ \\
\hline $\mathrm{HDL} \mathrm{mmol} / \mathrm{l}$ & $0.6 \pm 0.1$ & $0.9 \pm 0.1$ & $0.8 \pm 0.1$ & $0.8 \pm 0.1$ & $0.6 \pm 0.1$ & $1.2 \pm 0.1$ & $1.1 \pm 0.1$ & $\begin{array}{l}\mathrm{P}_{1-2^{2}, 3}>0.05 \\
\mathrm{P}_{4-6}<0.001 \\
\mathrm{P}^{-}<0.001\end{array}$ \\
\hline LDL mmol/l & $0.2 \pm 0.1$ & $2.5 \pm 0.1$ & $2.0 \pm 0.1$ & $0.2 \pm 0.1$ & $1.2 \pm 0.1$ & $4.0 \pm 0.5$ & $3.5 \pm 0.2$ & $\begin{array}{l}P_{1-2^{\prime}, 3}^{5}<0.05 \\
P_{4-6}<0.001 \\
P_{5.7}<0.001\end{array}$ \\
\hline CA & $1.1 \pm 0.1$ & $3.1 \pm 0.1$ & $2.6 \pm 0.1$ & $1.2 \pm 0.1$ & $2.5 \pm 0.3$ & $4.6 \pm 0.7$ & $4.0 \pm 0.3$ & $\begin{array}{l}\mathrm{P}_{1-2^{\prime}, 3}<0.05 \\
\mathrm{P}_{4-6}<0.05 \\
\mathrm{P}_{5-7}<0.05\end{array}$ \\
\hline
\end{tabular}

disorders in the development of fatty liver, the correlation of pro- and anti-inflammatory cytokines and cytokine index with the levels of TC, TG, LDL, and HDL was carried out. There was a statistically significant direct strong relationship between both cytokines and TC and LDL levels $(r=0.712$ and 0.744 , respectively, $p<0.001)$. A direct moderate relationship was demonstrated by IL-4 and TNF with TG $(r=0.402$ and 0.356 , respectively, $p<0.01$ ). The levels of IL-4 and TNF correlate statistically significantly with HDL ( $r$ $=0.536$ and 0.580 , respectively, $p<0.001$ ) .

Thus, a statistically significant positive correlation was found between hypercholesterolemia, TG, HDL, and LDL with TNF- $\alpha$ and IL-4 values in NAFLD developing under conditions of hypobaric hypoxia. In high altitude conditions, the role of both pro- and anti-inflammatory cytokines in the pathogenesis of fatty hepatosis increases, which should be taken into account in the management of such patients. Liver lipogenesis induced by fructose and hypoxia can cause liver inflammation and insulin resistance, with a tendency to decrease cytokines and lipid spectrum at 10 weeks at altitude in a pressure chamber.

\section{Conclusion}

In animals on a diet enriched with fructose under conditions of hypobaric hypoxia for 5 weeks, against the background of changes in the lipid spectrum (hypercholesterolemia, hypertriglyceridemia and dyslipidemia), the levels of both pro and antiinflammatory cytokines significantly increase, with a subsequent tendency to decrease at 10 weeks.

The cytokine index, which shows the ratio of pro-inflammatory to anti-inflammatory cytokines, in animals under hypobaric hypoxia on a diet enriched with fructose for 5 weeks is characterized by a higher value than in the low-mountain group on an identical diet, and at 10 weeks it is characterized by a decrease in relation to the low-mountain group.
Disorders of lipid metabolism (TC, TG, HDL, and $L D L$ ) in animals on a diet enriched with fructose are statistically significantly correlated with levels of TNF- $\alpha$ and IL-4.

\section{References}

1. Buzzetti E, Pinzani M, Tsochatzis EA. The multiple-hit pathogenesis of non-alcoholic fatty liver disease (NAFLD). Metabolism. 2016;65(8):1038-48. https://doi.org/10.1016/j. metabol.2015.12.012

PMid:26823198

2. Tilg $\mathrm{H}$, Diehl AM. Cytokines in alcoholic and nonalcoholic steatohepatitis. N Engl J Med. 2000;343(20):1467-76. https:// doi.org/10.1056/nejm200011163432007

PMid:11078773

3. Hotamisligil GS, Shargill NS, Spiegelman BM. Adipose expression of tumor necrosis factor-alpha: Direct role in obesitylinked insulin resistance. Science. 1993;259(5091):87-91. https://doi.org/10.1126/science.7678183

PMid:7678183

4. Koca SS, Bahcecioglu IH, Poyrazoglu OK, Ozercan IH, Sahin K, Ustundag B. The treatment with antibody of TNF-alpha reduces the inflammation, necrosis and fibrosis in the non-alcoholic steatohepatitis induced by methionine-and choline-deficient diet. Inflammation. 2008;31(2):91-8. https://doi.org/10.1007/ s10753-007-9053-z

PMid: 18066656

5. Braunersreuther V, Viviani GL, Mach F, Montecucco F. Role of cytokines and chemokines in non-alcoholic fatty liver disease. World J Gastroenterol. 2012;18(8):727-35. https://doi. org/10.3748/wjg.v18.i8.727

PMid:22371632

6. Akhmedov VA, Keruchenko AL. Involvement of cytokines in the mechanisms of the formation of non-alcoholic fatty liver disease. Vestnik NGU. 2012;10(1):76-80

7. Bespalova ID, Rjazanceva NV, Kaljuzhin VV, Afanas'eva DS, Murashev BJ, Osihov IA. Systemic inflammation in pathogenesis of metabolic syndrome and associated diseases. Siberian Med J. 2013;117(2):5-8.

8. Kondratyuk EA, Bodnar PN, Lisyanyy NI, Bel'skaya LN, Potapova Al. Cytokine status and subpopulation composition of peripheral blood lymphocytes in patients with Type 2 diabetes mellitus and non-alcoholic fatty liver disease. Endokrinologiya. 
2015;20(1):401-7. https://doi.org/10.1530/endoabs.37.ep500

9. Song K, Zhang Y, Ga Q, Bai Z, Ge RL. High-altitude chronic hypoxia ameliorates obesity-induced non-alcoholic fatty liver disease in mice by regulating mitochondrial and AMPK signaling. Life Sci. 2020;252:117633. https://doi.org/10.1016/j. Ifs. 2020.117633

PMid:32289432

10. Ackerman Z, Oron-Herman M, Grozovski M. Rosenthal T, Pappo O, Link G, et al. Fructose-induced fatty liver disease. Hypertension. 2005;45(5):1012-8. https://doi.org/10.1161/01. hyp.0000164570.20420.67

PMid:15824194

11. Dekker MJ, Su Q, Baker C, Rutledge AC, Adeli K. Fructose: A highly lipogenic nutrient implicated in insulin resistance, hepatic steatosis, and the metabolic syndrome. Am J Physiol Endocrinol Metab. 2010;299(5):E685-94. https://doi. org/10.1152/ajpendo.00283.2010

PMid:20823452

12. Zámbó V, Simon-Szabó L, Szelényi $P$, Kereszturi É, Bánhegyi G, Csala M. Lipotoxicity in the liver. World J Hepatol. 2013;5(10):550. https://doi.org/10.4254/wjh.v5.i10.550 PMid:24179614

13. Baffy G. Kupffer cells in non-alcoholic fatty liver disease: The emerging view. J Hepatol. 2009;51(1):212-23. https://doi. org/10.1016/j.jhep.2009.03.008

PMid:19447517

14. Galv S, O'Neill LA. Metabolic reprograming in macrophage polarization. Front Immunol. 2014;5:420.

PMid:25228902

15. Meerson FZ. Adaptation to High-Altitude Hypoxia. Physiology of Adaptation Processes, Nauka; 1986. p. 635.

Author Query???

AQ1: Kindly check the orcid link 